\title{
On the random dynamics of Volterra quadratic operators
}

\author{
U. U. JAMILOV $\dagger$, M. SCHEUTZOW $\ddagger$ and M. WILKE-BERENGUER $\ddagger$ \\ $\dagger$ Institute of Mathematics, National University of Uzbekistan, 29, Do'rmon Yo'li str., \\ 100125 Tashkent, Uzbekistan \\ (e-mail: jamilovu@yandex.ru) \\ $\ddagger$ Institut für Mathematik, MA 7-5, Fakultät II, Technische Universität Berlin, \\ Straße des 17. Juni 136, 10623 Berlin, Germany \\ (e-mail:ms@math.tu-berlin.de,wilkeber@math.tu-berlin.de)
}

(Received 30 January 2014 and accepted in revised form 12 March 2015)

\begin{abstract}
We consider random dynamical systems generated by a special class of Volterra quadratic stochastic operators on the simplex $S^{m-1}$. We prove that in contrast to the deterministic set-up the trajectories of the random dynamical system almost surely converge to one of the vertices of the simplex $S^{m-1}$, implying the survival of only one species. We also show that the minimal random point attractor of the system equals the set of all vertices. The convergence proof relies on a martingale-type limit theorem, which we prove in the appendix.
\end{abstract}

\section{Introduction}

The concept of a quadratic stochastic operator (QSO) and its application in a biological context were first established by Bernstein in [2]. Since then, the theory of QSOs has been further deepened motivated by their frequent occurrence in mathematical models of genetics, where QSOs serve as a tool for the study of dynamical properties and modelling; see [8-18, 20, 21, 23, 25, 26, 30-32]. While they were originally introduced as 'evolutionary operators' to describe the dynamics of gene frequencies for given laws of heredity in mathematical population genetics, QSOs and the dynamical systems they describe have become interesting objects of study in their own right from a purely mathematical point of view (see [23] for a comprehensive account).

In the description of the genetic evolution of large populations QSOs arise as follows: consider a population with $m \in \mathbb{N}$ different genetic types, where every individual in this population belongs to precisely one of the species $\llbracket m \rrbracket:=\{1,2, \ldots, m\}$. Let $\mathbf{x}^{0}=$ $\left(x_{1}^{0}, \ldots, x_{m}^{0}\right)$ be a probability distribution on $\llbracket m \rrbracket$ describing the relative frequencies of the genetic types within the whole population in the initial generation. Denote by $p_{i j, k}$ 
the conditional probability that two individuals of type $i$, respectively $j$, produce an offspring of type $k$ given they interbreed and assume that the population is large enough for frequency fluctuations to be neglectable. Presuming a free population, i.e. absence of sexual differentiation and the statistical independence of genotypes for breeding, the distribution $\mathbf{x}^{\prime}=\left(x_{1}^{\prime}, \ldots, x_{m}^{\prime}\right)$ of the (expected) gene frequencies in the next generation is given by

$$
x_{k}^{\prime}=\sum_{i, j=1}^{m} p_{i j, k} x_{i}^{0} x_{j}^{0}, \quad k \in \llbracket m \rrbracket .
$$

The association $\mathbf{x}^{0} \mapsto \mathbf{x}^{\prime}$ defines a map $V: S^{m-1} \rightarrow S^{m-1}$ called an evolutionary operator, where $S^{m-1}$ denotes the simplex of all distributions on $\llbracket m \rrbracket$. The population evolves by starting from an arbitrary frequency distribution $\mathbf{x}^{0}$, then passing to the state $\mathbf{x}^{\prime}=V\left(\mathbf{x}^{0}\right)$ in the next 'generation', then to the state $\mathbf{x}^{\prime \prime}=V\left(V\left(\mathbf{x}^{0}\right)\right)$, and so on. Thus, the evolution of gene frequencies in this population can be considered as a dynamical system

$$
\mathbf{x}^{0}, \quad \mathbf{x}^{\prime}=V\left(\mathbf{x}^{0}\right), \quad \mathbf{x}^{\prime \prime}=V^{2}\left(\mathbf{x}^{0}\right), \quad \mathbf{x}^{\prime \prime \prime}=V^{3}\left(\mathbf{x}^{0}\right), \ldots
$$

Note that $V$ as defined by (1.1) is a non-linear (quadratic) operator.

One of the main objects of study for dynamical systems and QSOs is the asymptotic behaviour of their trajectories, depending on the initial value. However, this has so far only been determined for certain particular subclasses of QSOs. One such subclass that arises naturally in the biological context is given by the additional restriction

$$
p_{i j, k}=0 \quad \text { if } k \notin\{i, j\}, i, j, k \in \llbracket m \rrbracket .
$$

These QSOs describe a reproductory behaviour where an offspring is a genetic copy of one of its parents and are called Volterra operators. The asymptotic behaviour of trajectories of this kind of QSOs was analysed in [10-17, 24, 27, 31, 32].

However, in the non-Volterra case (i.e. where condition (1.2) is violated), many questions remain open and there seems to be no general theory available. See [18] for a recent review of QSOs.

In all of the above-mentioned references the authors investigated deterministic trajectories of a QSO. However, it seems natural to consider a randomization of this procedure and explore the random dynamical system resulting from it. This can be done, e.g., by using a random iteration of operators of a given finite or countably infinite set of QSOs.

As a first step in this direction, we investigate the trajectories of a sequence of independent and identically distributed Volterra QSOs in the present work. We prove that for any initial point from the simplex of probability distributions the random trajectory converges almost surely to one of the vertices of the simplex. This is far from being obvious, since the set of Volterra QSOs considered may well contain operators that do not have this property and might, indeed, not converge at all. Furthermore, we show that the set of vertices of the simplex coincides with the minimal random point attractor of the corresponding random dynamical system.

Note that for the biological interpretation our results show that such a mechanism does not allow for coexistence but yields almost sure extinction of all but one species 
(Theorem 3.1). The corresponding results in the deterministic setting on the other hand cannot generally rule out coexistence in the long run (see e.g. Proposition 2.3(2)). Indeed, some of the QSOs included in the set we consider for the random setting, e.g. those studied in [32], model a very distinct deterministic behaviour. They describe a population where a species will come to the verge of extinction only to recover to the point where all other species are almost annihilated, after which the cycle repeats indefinitely, not yielding a stable situation.

The paper is organized as follows. In $\S 2$, we recall definitions and well-known results from the theory of Volterra QSOs and the definition of random QSOs. In §3, we define a special class of Volterra QSOs and show the almost sure convergence of the random iteration of these operators. Finally, in $\S 4$, we identify the minimal random point attractor of the resulting random dynamical system. In the appendix, we formulate and prove a martingale-type limit theorem, which we need for the proof of the main result of $\S 3$.

\section{Preliminaries and known results}

A quadratic stochastic operator (QSO) on $\llbracket m \rrbracket=\{1, \ldots, m\}$ is a mapping $V$ of the simplex

$$
S^{m-1}=\left\{\mathbf{x}=\left(x_{1}, \ldots, x_{m}\right) \in \mathbb{R}^{m}: x_{i} \geq 0, \forall i \in \llbracket m \rrbracket, \sum_{i=1}^{m} x_{i}=1\right\}
$$

into itself, of the form $V(\mathbf{x})=\mathbf{x}^{\prime} \in S^{m-1}$, where

$$
x_{k}^{\prime}=\sum_{i, j \in \llbracket m \rrbracket} p_{i j, k} x_{i} x_{j}, \quad k \in \llbracket m \rrbracket,
$$

and the $p_{i j, k}$ satisfy

$$
p_{i j, k}=p_{j i, k} \geq 0, \quad i, j, k \in \llbracket m \rrbracket, \quad \sum_{k=1}^{m} p_{i j, k}=1, \quad i, j \in \llbracket m \rrbracket .
$$

Note that we can assume the first condition in (2.3) without loss of generality (otherwise replace the inheritance coefficients by $\left.q_{i j, k}=\left(p_{i j, k}+p_{j i, k}\right) / 2, i, j, k \in \llbracket m \rrbracket\right)$.

The trajectory (orbit) $\left\{\mathbf{x}^{(n)}\right\}_{n \in \mathbb{N}_{0}}$ of $V$ for an initial value $\mathbf{x}^{(0)} \in S^{m-1}$ is defined by

$$
\mathbf{x}^{(n+1)}=V\left(\mathbf{x}^{(n)}\right)=V^{n+1}\left(\mathbf{x}^{(0)}\right), \quad n=0,1,2, \ldots
$$

The following notation will be used throughout this paper. We let int $S^{m-1}$ denote the interior and $\partial S^{m-1}$ the boundary of $S^{m-1}$, i.e.

$$
\text { int } S^{m-1}:=\left\{\mathbf{x} \in S^{m-1}: x_{1} x_{2} \cdots x_{m}>0\right\} \quad \text { and } \quad \partial S^{m-1}:=S^{m-1} \backslash \text { int } S^{m-1} .
$$

Furthermore, let $\mathbf{e}_{i}=\left(\delta_{1 i}, \delta_{2 i}, \ldots, \delta_{m i}\right)$ for $i=1,2, \ldots, m$ be the $i$ th vertex of the simplex $S^{m-1}$, where $\delta_{i j}$ is the Kronecker symbol. The set of limit points of the trajectory (2.4) is denoted by $\omega\left(\mathbf{x}^{(0)}\right)$.

A point $\mathbf{x} \in S^{m-1}$ is called a fixed point of $V$ if $V(\mathbf{x})=\mathbf{x}$. Note that our QSOs are continuous operators and that the simplex over a finite set is compact and convex, so that by the Brouwer fixed-point theorem there is always at least one fixed point. Further, if a trajectory generated by the QSO $V$ converges to some $\mathbf{x}$, then, by continuity, $\mathbf{x}$ is a fixed point. 
2.1. Volterra quadratic stochastic operators. Let $V$ be a quadratic stochastic operator on the simplex $S^{m-1}$.

Definition 2.1. The quadratic stochastic operator $V$ is called a Volterra operator if $p_{i j, k}=$ 0 for any $k \notin\{i, j\}, i, j, k \in \llbracket m \rrbracket$.

Evidently, for any Volterra QSO,

$$
p_{i i, i}=1 \quad \text { and } \quad p_{i k, k}+p_{i k, i}=1 \quad \text { for all } i, k \in \llbracket m \rrbracket, i \neq k .
$$

A Volterra QSO $V$ defined on $S^{m-1}$ therefore has the following form:

$$
(V(\mathbf{x}))_{k}=x_{k}^{2}+2 \sum_{i \in \llbracket m \rrbracket, i \neq k} p_{i k, k} x_{i} x_{k}, \quad k \in \llbracket m \rrbracket .
$$

PROPOSITION 2.2. [15] A QSO $V$ is a Volterra operator if and only if

$$
(V(\mathbf{x}))_{k}=x_{k}\left(1+\sum_{i=1}^{m} a_{k i} x_{i}\right),
$$

where $A=\left(a_{i j}\right)_{1}^{m}$ is a skew-symmetric matrix with $a_{k i}=2 p_{i k, k}-1$ for $i \neq k, a_{i i}=0$ and $\left|a_{i j}\right| \leq 1$. Here $i, j, k \in \llbracket m \rrbracket$.

The space of skew-symmetric matrices generating Volterra operators is parameterized by the cube $[-1,1]^{m(m-1) / 2}$. The extremal points of the cube are its vertices. The quadratic stochastic operator $V$ is called an extremal Volterra operator if the corresponding skew-symmetric matrix is a vertex of the cube, i.e. $a_{i j}=-1$ or 1 for any $i \neq j$.

It is evident that the total number of extremal Volterra QSO is equal to $2^{(m(m-1)) / 2}$.

PROPOSITION 2.3. [15] Let $V$ be a Volterra QSO. Then:

(1) $V$ is a homeomorphism on $S^{m-1}$;

(2) if $\mathbf{x}$ is not a fixed point of $V$, then $\omega(\mathbf{x}) \subset \partial S^{m-1}$.

PROPOSITION 2.4. For any Volterra operator $V$ and any $k \in \llbracket m \rrbracket$, we have

$$
(V(\mathbf{x}))_{k} \leq 2 x_{k} .
$$

Proof. The proof immediately follows from Proposition 2.2.

2.2. Random Volterra quadratic stochastic operators. In this subsection, we present the definition of a random Volterra quadratic stochastic operator in analogy to the more general concept of random QSOs introduced in [9]. Let $\mathcal{V}$ be the set of all Volterra quadratic stochastic operators defined on the unit simplex $S^{m-1}$. Since every QSO is represented by a cubic matrix $\left(p_{i j, k}\right)_{i, j, k \in \llbracket m \rrbracket}$, the set $\mathcal{V}$ is compactly embedded in $\mathbb{R}^{m^{3}}$. Let $\mathcal{H}$ be the Borel $\sigma$-algebra induced on the set $\mathcal{V}$.

Definition 2.5. Consider a probability space $(\Omega, \mathfrak{F}, \mathbb{P})$. Any measurable map $T: \Omega \rightarrow \mathcal{V}$ (i.e. such that $T^{-1}(\mathcal{H}) \subset \mathfrak{F}$ ) is called a random Volterra quadratic stochastic operator (RVQSO).

The notion of general random QSOs was introduced in [9] to study the related class of dyadic random quadratic stochastic operators in a random environment. 


\section{Main result}

For each $k \in \llbracket m \rrbracket$, define

$$
\mathcal{V}_{k}:=\left\{V \in \mathcal{V} \mid \forall \mathbf{x} \in S^{m-1}:(V(\mathbf{x}))_{k}=x_{k}^{2}\right\} .
$$

Note that $V \in \mathcal{V}_{k}$ holds if and only if the associated skew-symmetric matrix $A$ in Proposition 2.2 satisfies $a_{k i}=-1$ for all $i \neq k$. Therefore, the sets $\mathcal{V}_{k}, k \in \llbracket m \rrbracket$ are pairwise disjoint and non-empty. In fact, they even contain extremal Volterra operators.

THEOREM 3.1. Let $v$ be a probability distribution on $(V, \mathcal{H})$ such that $v_{i}:=v\left(\mathcal{V}_{i}\right)>0$ for all $i \in \llbracket m \rrbracket$. Consider a sequence $T_{1}, T_{2}, \ldots$ of independent and identically distributed $R V Q S O$ s in $\mathcal{V}$ such that the distribution of $T_{1}$ is given by $v$. Then, for any $\mathbf{x} \in S^{m-1}$,

$$
\mathbb{P}\left(\lim _{n \rightarrow \infty}\left(T_{n} \circ \cdots \circ T_{1}\right)(\mathbf{x}) \in\left\{\mathbf{e}_{1}, \ldots, \mathbf{e}_{m}\right\}\right)=1 .
$$

For $\varepsilon>0$, we denote by $U_{i}^{\varepsilon}=\left\{\mathbf{x} \in S^{m-1}: x_{j}<\varepsilon, j \in \llbracket m \rrbracket \backslash\{i\}\right\}$ the $\varepsilon$-neighbourhood of the vertex $\mathbf{e}_{i}, \quad i \in \llbracket m \rrbracket$, and set $U_{\varepsilon}=\bigcup_{i \in \llbracket m \rrbracket} U_{i}^{\varepsilon}$. Furthermore, we define $\Lambda:=$ $\left\{\mathbf{e}_{1}, \ldots, \mathbf{e}_{m}\right\}$ to be the set of vertices of $S^{m-1}$. The following Proposition 3.2 states that for any $\varepsilon>0$, there is a deterministic number $N \in \mathbb{N}$ of steps after which the probability of being close to one of the vertices, i.e. in $U_{\varepsilon}$, is bounded away from 0 uniformly with respect to the starting point $\mathbf{x} \in S^{m-1}$. Proposition 3.3 then in particular implies a positive probability of the trajectory converging to the corresponding vertex on this event. Since we can thus bound the probability of reaching $U_{\varepsilon}$ and subsequently converging to the closest vertex in $\Lambda$ away from 0 uniformly, the main result given in Theorem 3.1 then follows with a Borel-Cantelli-type argument.

PROPOSITION 3.2. Under the assumptions of Theorem 3.1, for each $\varepsilon>0$ there are $N \in \mathbb{N}$ and $q>0$ such that for every point $\mathbf{x} \in S^{m-1}$,

$$
\mathbb{P}\left(T_{N} \circ T_{N-1} \circ \cdots \circ T_{1}(\mathbf{x}) \in U_{\varepsilon}\right) \geq q .
$$

Proof. Let $\varepsilon>0$ and choose $r \in \mathbb{N}$ sufficiently large for $-2^{r}+(m-2) r<\log (\varepsilon) / \log (2)$ to hold. For a fixed starting point $\mathbf{x} \in S^{m-1}$, we will first construct a deterministic sequence $\bar{V}_{1}^{(1)}, \ldots, \bar{V}_{1}^{(r)}, \ldots, \bar{V}_{m-1}^{(1)}, \ldots, \bar{V}_{m-1}^{(r)} \in \mathcal{V}$ such that $\bar{V}_{m-1}^{(r)} \circ \cdots \circ \bar{V}_{m-1}^{(1)} \circ \cdots \circ$ $\bar{V}_{1}^{(r)} \circ \cdots \circ \bar{V}_{1}^{(1)}(\mathbf{x}) \in U_{\varepsilon}$ and then prove the probability of such an event to be bounded away from 0 uniformly in $\mathbf{x}$.

Define $j_{1}=j_{1}(\mathbf{x}) \in \llbracket m \rrbracket$ as the smallest index of a vertex corresponding to the maximal distance of $\mathbf{x}$ to $\Lambda$, i.e.

$$
\left\|\mathbf{x}-\mathbf{e}_{j_{1}}\right\|=\max _{j \in \llbracket m \rrbracket}\left\|\mathbf{x}-\mathbf{e}_{j}\right\|
$$

and $j_{1}$ is the smallest value for which this holds. We now recursively define a family of maps $j_{k}: \mathcal{V}^{r(k-1)} \rightarrow \llbracket m \rrbracket$ for $k=2, \ldots, m-1$ : set $J_{k}=J_{k}\left(V_{1}, \ldots, V_{r(k-1)}\right):=$ $\left\{j_{1}, j_{2}\left(V_{1}, \ldots, V_{r}\right), \ldots, j_{k}\left(V_{1}, \ldots, V_{r(k-1)}\right)\right\}$. Then define $j_{k+1}\left(V_{1}, \ldots, V_{r k}\right) \in \llbracket m \rrbracket \backslash$ $J_{k}$ to be the smallest index such that

$$
\left\|V_{r k} \circ \cdots \circ V_{1}(\mathbf{x})-\mathbf{e}_{j_{k+1}\left(V_{1}, \ldots, V_{r k}\right)}\right\|=\max _{j \in \llbracket m \rrbracket \backslash J_{k}\left(V_{1}, \ldots, V_{r(k-1)}\right)}\left\|V_{r k} \circ \cdots \circ V_{1}(\mathbf{x})-\mathbf{e}_{j}\right\| .
$$




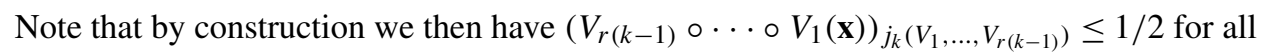
$k=2, \ldots, m-1$ and any choice of $V_{1}, \ldots, V_{r(k-1)} \in \mathcal{V}$.

Proceed to choose $\bar{V}_{1}^{(1)}, \ldots, \bar{V}_{1}^{(r)}, \ldots, \bar{V}_{m-1}^{(1)}, \ldots, \bar{V}_{m-1}^{(r)} \in \mathcal{V}$ such that

$$
\bar{V}_{k}^{(1)}, \ldots, \bar{V}_{k}^{(r)} \in \mathcal{V}_{j_{k}\left(\bar{V}_{1}^{(1)}, \ldots, \bar{V}_{k-1}^{(r)}\right)}, \quad k \in \llbracket m-1 \rrbracket .
$$

With this choice, we obtain the following estimates for every $k \in \llbracket m-1 \rrbracket$ :

$$
\begin{aligned}
& \left(\bar{V}_{m-1}^{(r)} \circ \cdots \circ \bar{V}_{m-1}^{(1)} \circ \cdots \circ \bar{V}_{1}^{(r)} \circ \cdots \circ \bar{V}_{1}^{(1)}(\mathbf{x})\right)_{j_{k}\left(\bar{V}_{1}^{(1)}, \ldots, \bar{V}_{k-1}^{(r)}\right)} \\
& \quad=\left(\bar{V}_{m-1}^{(r)} \circ \cdots \circ \bar{V}_{k+1}^{(1)}\left(\bar{V}_{k}^{(r)} \circ \cdots \circ \bar{V}_{k}^{(1)}\left(\bar{V}_{k-1}^{(r)} \circ \cdots \circ \bar{V}_{1}^{(1)}(\mathbf{x})\right)\right)\right)_{j_{k}\left(\bar{V}_{1}^{(1)}, \ldots, \bar{V}_{k-1}^{(r)}\right)} \\
& \quad \leq 2^{r(m-1-k)}\left(\bar{V}_{k}^{(r)} \circ \cdots \circ \bar{V}_{k}^{(1)}\left(\bar{V}_{k-1}^{(r)} \circ \cdots \circ \bar{V}_{1}^{(1)}(\mathbf{x})\right)\right) j_{k}\left(\bar{V}_{1}^{(1)}, \ldots, \bar{V}_{k-1}^{(r)}\right) \\
& \quad \leq 2^{r(m-1-k)}\left(\bar{V}_{k-1}^{(r)} \circ \cdots \circ \bar{V}_{1}^{(1)}(\mathbf{x})\right)^{\left(2^{r}\right)}{ }_{j k}^{\left(\bar{V}_{1}^{(1)}, \ldots, \bar{V}_{k-1}^{(r)}\right)} \\
& \quad \leq 2^{r(m-1-k)}\left(\frac{1}{2}\right)^{\left(2^{r}\right)}=2^{r(m-1-k)-2^{r}} \leq 2^{r(m-2)-2^{r}}<\varepsilon,
\end{aligned}
$$

where we used Proposition 2.4 in the first inequality and (3.1) in the second. This implies

$$
\bar{V}_{m-1}^{(r)} \circ \cdots \circ \bar{V}_{1}^{(1)}(\mathbf{x}) \in U_{\varepsilon}^{j_{*}} \subset U_{\varepsilon}
$$

if we denote by $j_{*}$ the unique element of $\llbracket m \rrbracket \backslash J_{m-1}\left(\bar{V}_{1}^{(1)}, \ldots, \bar{V}_{m-1}^{(r)}\right)$. Observe that the assumption of independence allows us to estimate the probability of such a suitable choice of operators satisfying (3.2), which in turn is a lower bound for the value we want to bound away from 0 : if we define $\underline{v}:=\min \left\{v_{1}, \ldots, v_{m}\right\}$, then

$$
\begin{aligned}
\mathbb{P}\left(T_{r}(m-1)\right. & \left.\circ \cdots \circ T_{1}(\mathbf{x}) \in U_{\varepsilon}\right) \\
& \geq \sum_{i_{2}=1}^{m} \ldots \sum_{i_{m-1}=1}^{m} \mathbb{P}\left(T_{(m-1) r}, \ldots, T_{(m-2) r+1} \in \mathcal{V}_{i_{m-1}}, j_{m-1}\left(T_{1}, \ldots, T_{(m-2) r}\right)\right. \\
& \left.=i_{m-1}, \ldots, T_{2 r}, \ldots, T_{r+1} \in \mathcal{V}_{i_{2}}, j_{2}\left(T_{1}, \ldots, T_{r}\right)=i_{2}, T_{r}, \ldots, T_{1} \in \mathcal{V}_{1}\right) \\
& =\sum^{m} \ldots \sum_{i_{m-1}=1}^{m} \mathbb{P}\left(T_{(m-1) r}, \ldots, T_{(m-2) r+1} \in \mathcal{V}_{i_{m-1}} \mid j_{m-1}\left(T_{1}, \ldots, T_{(m-2) r}\right)\right. \\
& =i_{m-1}, \ldots, T_{2 r}, \ldots, T_{r+1} \in \mathcal{V}_{i_{2}}, j_{2}\left(T_{1}, \ldots, T_{r}\right) \\
& \left.=i_{2}, T_{r}, \ldots, T_{1} \in \mathcal{V}_{1}\right) \mathbb{P}\left(j_{m-1}\left(T_{1}, \ldots, T_{(m-2) r}\right)\right. \\
& \left.=i_{m-1}, \ldots, T_{2 r}, \ldots, T_{r+1} \in \mathcal{V}_{i_{2}}, j_{2}\left(T_{1}, \ldots, T_{r}\right)=i_{2}, T_{r}, \ldots, T_{1} \in \mathcal{V}_{1}\right) \\
& =\sum_{i_{2}=1}^{m} \ldots \sum_{i_{m-1}=1}^{m} \underbrace{\mathbb{P}\left(T_{(m-1) r}, \ldots, T_{(m-2) r+1} \in \mathcal{V}_{i_{m-1}}\right)}_{=v_{i_{m-1}} \geq \underline{v}^{r}} \mathbb{P}\left(j_{m-1}\left(T_{1}, \ldots, T_{(m-2) r}\right)\right. \\
& \left.=i_{m-1}, \ldots, T_{2 r}, \ldots, T_{r+1} \in \mathcal{V}_{i_{2}}, j_{2}\left(T_{1}, \ldots, T_{r}\right)=i_{2}, T_{r}, \ldots, T_{1} \in \mathcal{V}_{1}\right) \\
& \geq \underline{v}^{r} \sum_{i_{2}=1}^{m} \ldots \sum_{i_{m-2}=1}^{m} \mathbb{P}\left(T_{(m-2) r}, \ldots, T_{(m-3) r+1}\right. \\
\in & \left.\mathcal{V}_{i_{m-2}}, \ldots, T_{2 r}, \ldots, T_{r+1} \in \mathcal{V}_{i_{2}}, j_{2}\left(T_{1}, \ldots, T_{r}\right)=i_{2}, T_{r}, \ldots, T_{1} \in \mathcal{V}_{1}\right) .
\end{aligned}
$$


Iterating this argument yields

$$
\mathbb{P}\left(T_{r(m-1)} \circ \cdots \circ T_{1}(\mathbf{x}) \in U_{\varepsilon}\right) \geq \underline{v}^{r(m-1)}>0 .
$$

Since this does not depend on our initial choice of $\mathbf{x}$ any more, we have proven the claim with $q=\underline{v}^{(m-1) r}$ and $N=r(m-1)$.

In order to analyse the convergence, consider a sequence $\left(T_{n}\right)_{n \in \mathbb{N}}$ of random QSOs as in Theorem 3.1 and let $X$ denote a random variable taking values in $\operatorname{int} S^{m-1}$ that is independent of the sequence and such that $\mathbb{E}[|\log (X)|]<\infty$. Define a filtration $\left(\mathcal{F}_{n}\right)_{n \in \mathbb{N}_{0}}$ by $\mathcal{F}_{n}:=\sigma\left(X, T_{1}, \ldots, T_{n}\right)$ for $n \in \mathbb{N}_{0}$. We introduce the abbreviation $\hat{T}_{n}:=T_{n} \circ \cdots \circ T_{1}$ and use this to define

$$
Z_{n}^{i}:=\log \left(\left(\hat{T}_{n} X\right)_{i}\right)
$$

Note that, by Proposition 2.2, $\hat{T}_{n} X \in \operatorname{int} S^{m-1}$ for all $n \in \mathbb{N}$ and thus (3.3) is well defined.

We would like the increments of this process to be (at least) integrable, but since this is not necessarily the case we define a new process $\left(Y_{n}^{i}\right)_{n \in \mathbb{N}_{0}}$ in the following way: choose $d>\max \left\{\log (m), \max _{i \in \llbracket m \rrbracket}\left\{1 / \nu_{i}\right\} \log (2)\right\}$ and set

$$
\begin{aligned}
Y_{0}^{i} & :=Z_{0}^{i}=\log \left(X^{i}\right), \\
Y_{n+1}^{i}-Y_{n}^{i} & := \begin{cases}Z_{n+1}^{i}-Z_{n}^{i} & \text { if } Z_{n+1}^{i}-Z_{n}^{i} \geq-d, \\
-d & \text { otherwise. }\end{cases}
\end{aligned}
$$

Then we know that for all $\omega \in \Omega, Z_{n}^{i}(\omega) \leq Y_{n}^{i}(\omega)$.

Proposition 3.3. For $D:=\min _{i \in \llbracket m \rrbracket}\left\{v_{i} d-\log (2)\right\}>0$, we have for every $j \in \llbracket m \rrbracket$

$$
\mathbb{P}\left(\forall i \in \llbracket m \rrbracket \backslash\{j\}: \liminf _{n \rightarrow \infty}-\frac{1}{n} Y_{n}^{i} \geq D \mid \forall i \in \llbracket m \rrbracket \backslash\{j\}: \forall n \in \mathbb{N}: Y_{n}^{i} \leq-d\right)=1 .
$$

Moreover, for every $\theta>0$ and every $b \in \mathbb{R}$, there exists an $s>0$ such that

$$
\mathbb{P}\left(\exists j \in \llbracket m \rrbracket \forall i \in \llbracket m \rrbracket \backslash\{j\} \forall n \in \mathbb{N}: Y_{n}^{i}<b \mid \mathcal{F}_{0}\right) \geq 1-\theta \text { on }\left\{X \in \bar{U}_{s}\right\} \mathbb{P} \text {-a.s., }
$$

where $\bar{U}_{s}:=\left\{\mathbf{x} \in S^{m-1}: \exists j \in \llbracket m \rrbracket \forall i \neq j: x_{j} \leq \varepsilon\right\}$.

Proof. Note that the increments of $\left(Y_{n}^{i}\right)_{n \in \mathbb{N}_{0}}$ are integrable. Thus, we can calculate

$$
\begin{aligned}
\mathbb{E}\left[Y_{n+1}^{i}\right. & \left.-Y_{n}^{i} \mid \mathcal{F}_{n}\right]=\mathbb{E}\left[\log \left(\frac{T_{n+1}\left(\hat{T}_{n}(X)\right)_{i}}{\hat{T}_{n}(X)_{i}}\right) \vee(-d) \mid \mathcal{F}_{n}\right] \\
& =\int_{\mathcal{V}} \log \left(\frac{V\left(\hat{T}_{n}(X)\right)_{i}}{\hat{T}_{n}(X)_{i}}\right) \vee(-d) d v(V) \\
& =\int_{\mathcal{V}_{i}} \log \underbrace{\left(\frac{V\left(\hat{T}_{n}(X)\right)_{i}}{\hat{T}_{n}(X)_{i}}\right)}_{=\hat{T}_{n}(X)_{i}} \vee(-d) d v(V)+\int_{\mathcal{V} \backslash \mathcal{V}_{i}} \log \underbrace{\left(\frac{V\left(\hat{T}_{n}(X)\right)_{i}}{\hat{T}_{n}(X)_{i}}\right)}_{\leq 2 \text { by Proposition } 2.4} \vee(-d) d v(V) \\
& \leq v_{i}\left(\log \left(\hat{T}_{n}(X)_{i}\right) \vee(-d)\right)+\log (2) \\
& =v_{i}\left(Z_{n}^{i} \vee(-d)\right)+\log (2) \\
& \leq-v_{i} d+\log (2) \leq-D \text { on }\left\{Z_{n}^{i} \leq-d\right\} \text { and therefore also on }\left\{Y_{n}^{i} \leq-d\right\}
\end{aligned}
$$


and

$$
\begin{aligned}
& \mathbb{E}\left[\left(Y_{n+1}^{i}-\mathbb{E}\left[Y_{n+1}^{i} \mid \mathcal{F}_{n}\right]\right)^{2} \mid \mathcal{F}_{n}\right]=\mathbb{E}\left[\left(Y_{n+1}^{i}-Y_{n}^{i}-\mathbb{E}\left[Y_{n+1}^{i}-Y_{n}^{i} \mid \mathcal{F}_{n}\right]\right)^{2} \mid \mathcal{F}_{n}\right] \\
& =\mathbb{E}\left[\left(Y_{n+1}^{i}-Y_{n}^{i}\right)^{2} \mid \mathcal{F}_{n}\right]-\mathbb{E}\left[\left(\mathbb{E}\left[Y_{n+1}^{i}-Y_{n}^{i} \mid \mathcal{F}_{n}\right]\right)^{2} \mid \mathcal{F}_{n}\right] \\
& \leq \mathbb{E}\left[(\underbrace{\left(Y_{n+1}^{i}-Y_{n}^{i}\right)^{+}}_{\leq \log (2)})^{2} \mid \mathcal{F}_{n}\right]+\mathbb{E}\left[(\underbrace{\left(Y_{n+1}^{i}-Y_{n}^{i}\right)^{-}}_{\leq d})^{2} \mid \mathcal{F}_{n}\right] \\
& \leq(\log (2))^{2}+d^{2} \quad \mathbb{P} \text {-a.s. }
\end{aligned}
$$

This allows us to apply Proposition A.1 (Appendix), yielding

$$
\mathbb{P}\left(\liminf _{n \rightarrow \infty}-\frac{1}{n} Y_{n}^{i} \geq D \mid \forall n \in \mathbb{N}: Y_{n}^{i} \leq-d\right)=1
$$

and that for every $\theta>0$ and every $b \in \mathbb{R}$ there exists an $r_{i} \in \mathbb{R}$ such that

$$
\mathbb{P}\left(\forall n \in \mathbb{N}: Y_{n}^{i}<b \mid \mathcal{F}_{0}\right) \geq 1-\frac{1}{m-1} \theta \text { on }\left\{Y_{0}^{i} \leq r_{i}\right\}=\left\{\log \left(X^{i}\right) \leq r_{i}\right\} .
$$

From (3.6), we obtain for every $j \in \llbracket m \rrbracket$

$$
\mathbb{P}\left(\forall i \in \llbracket m \rrbracket \backslash\{j\}: \liminf _{n \rightarrow \infty}-\frac{1}{n} Y_{n}^{i} \geq D \mid \forall i \in \llbracket m \rrbracket \backslash\{j\} \forall n \in \mathbb{N}: Y_{n}^{i} \leq-d\right)=1 .
$$

With $s:=\min _{i=1, \ldots, n}\left\{\exp \left(r_{i}\right)\right\}$ for any $j \in \llbracket m \rrbracket$, (3.7) implies that

$$
\mathbb{P}\left(\forall i \in \llbracket m \rrbracket \backslash\{j\} \forall n \in \mathbb{N}: Y_{n}^{i}<b \mid \mathcal{F}_{0}\right) \geq 1-\theta \text { on }\left\{X \in \bar{U}_{s}^{j}\right\}=\bigcap_{i \in \llbracket m \rrbracket \backslash\{j\}}\left\{X^{i} \leq s\right\}
$$

and thus

$$
\mathbb{P}\left(\exists j \in \llbracket m \rrbracket \forall i \in \llbracket m \rrbracket \backslash\{j\}: \forall n \in \mathbb{N}: Y_{n}^{i}<b \mid \mathcal{F}_{0}\right) \geq 1-\theta \text { on }\left\{X \in \bar{U}_{s}\right\} .
$$

Proof of Theorem 3.1. Recall the definitions of $D$ and $d$ from above. Note that by Proposition 2.2, for any $k \in \llbracket m \rrbracket$ and every Volterra operator $V, x_{k} \neq 0$ if and only if $(V \mathbf{x})_{k} \neq 0$. Thus, by disregarding the zero entries, starting on $\partial S^{m-1}$ can be interpreted as starting and considering the same problem on the interior of a lower-dimensional simplex. Therefore, without loss of generality, we can assume $\mathbf{x} \in \operatorname{int} S^{m-1}$. Let $\theta \in(0,1)$ be arbitrary and, setting $b:=-d$, choose $s$ as in Proposition 3.3. For $\varepsilon:=\min \{s, 1 / m\}$, let $N$ and $q$ be as in Proposition 3.2.

We begin by defining the objects we will need for the proof. Define the stopping time

$$
\tau_{1}:=\inf \left\{n N \mid \exists j \in \llbracket m \rrbracket \forall i \in \llbracket m \rrbracket \backslash\{j\}: Z_{n N}^{i}<\log (\varepsilon)\right\}=\inf \left\{n N \mid \hat{T}_{n N}(\mathbf{x}) \in U_{\varepsilon}\right\} .
$$

Proposition 3.2 shows that $\tau_{1}$ is almost surely finite. Set $J_{1}:=\min \left\{j \in \llbracket m \rrbracket \mid \hat{T}_{\tau_{1}}(\mathbf{x}) \in U_{\varepsilon}^{j}\right\}$. Now, for every index $i \neq J_{1}$, we start the cut-off version $\left(Y_{n}^{\tau_{1}}\right)_{n \in \mathbb{N}_{0}}$ of our process given by

$$
\begin{aligned}
Y_{0}^{\tau_{1}, i} & :=\log (\varepsilon) \geq Z_{\tau_{1}}^{i}, \\
Y_{n+1}^{\tau_{1}, i}-Y_{n}^{\tau_{1}, i} & := \begin{cases}Z_{\tau_{1}+n+1}^{i}-Z_{\tau_{1}+n}^{i} & \text { if } Z_{\tau_{1}+n+1}^{i}-Z_{\tau_{1}+n}^{i} \geq-d, \\
-d & \text { otherwise. }\end{cases}
\end{aligned}
$$

for all $n \in \mathbb{N}_{0}$ and use this to define the stopping time

$$
\sigma_{1}:=\inf \left\{n>\tau_{1} \mid \exists i \neq J_{1}: Y_{n}^{\tau_{1}, i} \geq-d\right\} .
$$


Here, $J_{1}$ and $\sigma_{1}$ are well defined since $\tau_{1}<\infty \mathbb{P}$-almost surely. Recursively, then define

$$
\begin{aligned}
\tau_{k+1} & :=\inf \left\{n N>\sigma_{k} \mid \exists j \in \llbracket m \rrbracket: \forall i \in \llbracket m \rrbracket \backslash\{j\}: Z_{n N}^{i}<\log (\varepsilon)\right\} \\
& =\inf \left\{n N>\sigma_{k} \mid \hat{T}_{n N}(\mathbf{x}) \in U_{\varepsilon}\right\}, \\
J_{k+1} & :=\min \left\{j \in \llbracket m \rrbracket \mid \hat{T}_{\tau_{k+1}}(\mathbf{x})_{\tau_{k+1}} \in U_{\varepsilon}^{j}\right\}, \\
Y_{0}^{\tau_{k+1}, i} & :=\log (s) \geq Z_{\tau_{k+1}}^{i}, \\
Y_{n+1}^{\tau_{k+1}, i}-Y_{n}^{\tau_{k+1}, i} & := \begin{cases}Z_{\tau_{k+1}+n+1}^{i}-Z_{\tau_{k+1}+n}^{i} & \text { if } Z_{\tau_{k+1}+n+1}^{i}-Z_{\tau_{k+1}+n}^{i} \geq-d, \\
-d & \text { otherwise, }\end{cases} \\
\sigma_{k+1}: & =\inf \left\{n>\tau_{k+1} \mid \exists i \neq J_{k+1}: Y_{n}^{\tau_{k+1}, i} \geq-d\right\}
\end{aligned}
$$

for $i \neq J_{k+1}, n \in \mathbb{N}_{0}$.

Note that, on $\left\{\sigma_{k}=\infty\right\}$, we have the existence of a $j \in \llbracket m \rrbracket$ such that for all other $i \in \llbracket m \rrbracket \backslash\{j\}: Y_{n}^{\tau_{k}, i}<-d$ holds, which, by Proposition 3.3 and its definition, implies that $\lim _{n \rightarrow \infty} Z_{n}^{i}=-\infty$. This is, however, equivalent to $\lim _{n \rightarrow \infty} \hat{T}(\mathbf{x}) \in \Lambda$, our desired result.

Of course, since some of the above are only well defined when the corresponding stopping times are finite, we begin by considering the probabilities of these events. Again, by Proposition 3.2, we know that $\mathbb{P}\left(\tau_{k+1}<\infty \mid \mathcal{F}_{\sigma_{k}}\right)=1$ on $\left\{\sigma_{k}<\infty\right\}$. Furthermore, since $\left\{\tau_{k}<\infty\right\} \subset\left\{\hat{T}_{\tau_{k}}(\mathbf{x}) \in U_{\varepsilon}\right\}$, we know that

$$
\begin{aligned}
\mathbb{P}\left(\sigma_{k}=\infty \mid \mathcal{F}_{\tau_{k}}\right) & =\mathbb{P}\left(\exists j \in \llbracket m \rrbracket: \forall i \in \llbracket m \rrbracket \backslash\{j\}: \forall n \in \mathbb{N}_{0}: Y_{n}^{\tau_{k}, i}<-d \mid \mathcal{F}_{\tau_{k}}\right) \\
& \geq 1-\theta
\end{aligned}
$$

on $\left\{\tau_{k}<\infty\right\} \mathbb{P}$-almost surely by Proposition 3.3 and therefore $\mathbb{P}\left(\sigma_{k}<\infty \mid \mathcal{F}_{\tau_{k}}\right) \leq \theta$ on $\left\{\tau_{k}<\infty\right\}$. Combining the results above, we see that for every $k \in \mathbb{N}$ we have $\mathbb{P}\left(\sigma_{k}<\infty \mid\right.$ $\left.\mathcal{F}_{\sigma_{k-1}}\right) \leq \theta$ on $\left\{\sigma_{k-1}<\infty\right\}$, which we can use to conclude that

$$
\begin{aligned}
\mathbb{P}\left(\sigma_{k}<\infty\right) & =\mathbb{P}\left(\sigma_{k}<\infty, \ldots, \sigma_{1}<\infty\right) \\
& =\mathbb{E}(\underbrace{\mathbb{P}\left(\sigma_{k}<\infty \mid \mathcal{F}_{\sigma_{k-1}}\right)}_{\leq \theta} \mathbb{1}_{\left\{\sigma_{k-1}<\infty\right\}} \cdots \mathbb{1}_{\left\{\sigma_{1}<\infty\right\}}) \\
& \leq \theta \mathbb{P}\left(\sigma_{k-1}<\infty, \ldots, \sigma_{1}<\infty\right) \leq \theta^{k},
\end{aligned}
$$

iterating the argument used in the last step. Therefore, $\sum_{k \in \mathbb{N}} \mathbb{P}\left(\sigma_{k}<\infty\right)<\infty$, which, by the first Borel-Cantelli lemma, implies that $\mathbb{P}\left(\exists k \in \mathbb{N}: \sigma_{k}=\infty\right)=1$. Since we chose the $\left\{\forall i \in \llbracket m \rrbracket \backslash\{j\}: \forall n \in \mathbb{N}: Y_{n}^{i}<-d\right\}_{j \in \llbracket m \rrbracket}$ to be disjoint, by Proposition 3.3 we know that

$$
\mathbb{P}\left(\exists j \in \llbracket m \rrbracket \forall i \in \llbracket m \rrbracket \backslash\{j\}: \lim _{n \rightarrow \infty} Y_{n}^{\tau_{k}, i}=-\infty \mid \sigma_{k}=\infty\right)=1
$$

and can conclude that

$$
\begin{aligned}
1 & =\mathbb{P}\left(\exists k \in \mathbb{N} \exists j \in \llbracket m \rrbracket \forall i \in \llbracket m \rrbracket \backslash\{j\}: \lim _{n \rightarrow \infty} Y_{n}^{\tau_{k}, i}=-\infty\right) \\
& \leq \mathbb{P}\left(\exists j \in \llbracket m \rrbracket \forall i \in \llbracket m \rrbracket \backslash\{j\}: \lim _{n \rightarrow \infty} Z_{n}^{i}=-\infty\right) \\
& \leq \mathbb{P}\left(\lim _{n \rightarrow \infty} \hat{T}_{n}(\mathbf{x}) \in \Lambda\right),
\end{aligned}
$$

which completes the proof of Theorem 3.1. 
Remark 3.4. In this context, one can examine the (random) sets of sites converging to a specific vertex in $\Lambda$, i.e.

$$
M_{i}:=\left\{\mathbf{x} \in S^{m-1} \mid \lim _{n \rightarrow \infty} \hat{T}_{n}(\mathbf{x})=\mathbf{e}_{i}\right\}, \quad i \in \llbracket m \rrbracket .
$$

Since, by Proposition 2.4, every vertex in $\Lambda$ is a fixed point for any operator in $\mathcal{V}$, these sets are non-empty. Proposition 3.3, however, in addition allows us to conclude the almost sure existence of points $\mathbf{x} \in M_{i} \backslash\left\{\mathbf{e}_{i}\right\}$ for every $i \in \llbracket m \rrbracket$.

\section{Random attractors}

In this section, we recall the concept of a random attractor of a random dynamical system (RDS) and show that the RDS generated by the sequence of random operators in Theorem 3.1 has the set $\Lambda=\left\{\mathbf{e}_{1}, \ldots, \mathbf{e}_{m}\right\}$ as a minimal random point attractor. There exist a number of different concepts of random attractors, some of which we will introduce below. We restrict our attention to the discrete-time setting.

Let $(E, d)$ be a separable, complete metric space and denote its Borel- $\sigma$-field by $\mathcal{E}$. The following definition can be found in [1].

\section{Definition 4.1.}

(a) $\left(\Omega, \mathcal{F}, \mathbb{P},\left(\vartheta_{n}\right)_{n \in \mathbb{Z}}\right)$ is called a metric dynamical system $(M D S)$ if $(\Omega, \mathcal{F}, \mathbb{P})$ is a probability space and the family of maps $\left\{\vartheta_{n}: \Omega \rightarrow \Omega, n \in \mathbb{Z}\right\}$ satisfies:

(i) the mapping $\omega \mapsto \vartheta_{n}(\omega)$ is measurable for each $n \in \mathbb{Z}$;

(ii) $\vartheta_{m+n}=\vartheta_{m} \circ \vartheta_{n}$ for every $m, n \in \mathbb{Z}$ and $\vartheta_{0}=\operatorname{Id}_{\Omega}$;

(iii) $\vartheta_{1}$ preserves the measure $\mathbb{P}$ (and thus this holds for all $\vartheta_{n}, n \in \mathbb{Z}$ ).

(b) A random dynamical system $(R D S)$ on the measurable space $(E, \mathcal{E})$ over the MDS $\left(\Omega, \mathcal{F}, P,\left(\vartheta_{n}\right)\right)$ with time $\mathbb{N}_{0}$ is a mapping

$$
\varphi: \mathbb{N}_{0} \times E \times \Omega \rightarrow E, \quad(n, \mathbf{x}, \omega) \mapsto \varphi(n, \mathbf{x}, \omega)
$$

with the following properties:

(i) for each $n \in \mathbb{N}_{0}, \varphi(n, \cdot, \cdot)$ is $(\mathcal{E} \otimes \mathcal{F}, \mathcal{E})$-measurable;

(ii) for all $m, n \in \mathbb{N}_{0}$,

$$
\varphi(m+n, \omega)=\varphi\left(m, \vartheta_{n} \omega\right) \circ \varphi(n, \omega) \quad \text { for all } \omega \in \Omega
$$

and $\varphi(0, \omega)=\operatorname{Id}_{E}$ for all $\omega \in \Omega$.

The $\operatorname{RDS} \varphi$ is called continuous if, in addition:

(iii) the mapping $\mathbf{x} \mapsto \varphi(n, \mathbf{x}, \omega)$ is continuous for all $(n, \omega) \in \mathbb{N}_{0} \times \Omega$.

The following definition of a global attractor is (essentially) due to Crauel and Flandoli [7], while point attractors were introduced in [5].

Definition 4.2. Let $\varphi$ be an $\operatorname{RDS}$ on $E$ over the $\operatorname{MDS}\left(\Omega, \mathcal{F}, \mathbb{P},\left(\vartheta_{n}\right)_{n \in \mathbb{N}_{0}}\right)$. Let $\mathcal{B} \subset 2^{E}$ be an arbitrary subset of the power set of $E$. A family of sets $A(\omega) \in 2^{E}, \omega \in \Omega$ is called a $\mathcal{B}$-attractor for $\varphi$ if:

- $\quad A$ is a compact random set (i.e. $A(\omega)$ is non-empty and compact for every $\omega \in \Omega$ and $\omega \mapsto d(\mathbf{x}, A(\omega))$ is measurable for every $\mathbf{x} \in E)$; 
- $\quad A$ is strictly $\varphi$-invariant, that is, there exists a set $\Omega_{0}$ of full measure such that $\varphi(n, \omega)(A(\omega))=A\left(\vartheta_{n} \omega\right)$ for all $n \in \mathbb{N}_{0}, \omega \in \Omega_{0}$;

- $\quad \lim _{n \rightarrow \infty} \sup _{\mathbf{x} \in B} d\left(\varphi\left(n, \vartheta_{-n} \omega\right)(\mathbf{x}), A(\omega)\right)=0$ almost surely for every $B \in \mathcal{B}$.

In particular, a $\mathcal{B}$-attractor is called:

- a global attractor in case that $\mathcal{B}$ is the set of all compact subsets of $E$;

- a point attractor in case that $\mathcal{B}$ is the set of all singletons $\{\{\mathbf{x}\}, \mathbf{x} \in E\}$ (or, equivalently, the set of all finite subsets of $E$ ).

A random attractor as introduced in the previous definition is often called a strong attractor or a pullback attractor as opposed to a weak attractor, for which the almost sure convergence is relaxed to convergence in probability. One can argue that weak attractors occur more naturally than strong ones (see e.g. [3]) (but proving the existence of a strong attractor is of course a stronger statement). Sometimes the word compact is replaced by bounded in the definition of a global attractor. While a global attractor, if it exists, is always unique (up to sets of measure zero, see [4]) this is not true for a point attractor (Theorem 4.3 below provides an example). We call a point attractor $A(\omega)$ minimal if, for every other point attractor $\tilde{A}(\omega)$, we have $A(\omega) \subseteq \tilde{A}(\omega)$ for almost all $\omega \in \Omega$. Under mild assumptions, existence of a point attractor implies the existence of a minimal point attractor (see [5, Remark 3.5(iii)]). Clearly, each global attractor is also a point attractor but the converse is not necessarily true (again, Theorem 4.3 below provides an example). Note that a comparison between different concepts of a random attractor has been performed in a special case in [28] and criteria for strong and weak random attractors have been established in [6].

We are now ready to apply the concepts to the system introduced in the previous sections. We assume that all assumptions in Theorem 3.1 hold. As the basic probability space $(\Omega, \mathcal{F}, \mathbb{P})$, we take $(\Omega, \mathcal{F}, \mathbb{P}):=(\mathcal{V}, v)^{\mathbb{Z}}$ (where $\mathcal{V}$ is equipped with the $\sigma$-field of all subsets of $\mathcal{V})$. Further, we define $\left(\vartheta_{n}(\omega)\right)_{m}=\omega_{m+n}, m, n \in \mathbb{Z}$. Then $\left(\Omega, \mathcal{F}, \mathbb{P},\left(\vartheta_{n}\right)_{n \in \mathbb{N}_{0}}\right)$ is a metric dynamical system and $\varphi(n, \omega, \mathbf{x}):=\omega_{n} \circ \cdots \circ$ $\omega_{1}(\mathbf{x}), n \in \mathbb{N}_{0}, \mathbf{x} \in S^{m-1}$ defines an $S^{m-1}$-valued continuous RDS. Since $S^{m-1}$ is compact and all $V \in \mathcal{V}$ are homeomorphisms, it follows that $A(\omega):=S^{m-1}$ is the random attractor of $\varphi$. It turns out that $A$ is however not the minimal point attractor.

THEOREM 4.3. In the set-up above, the set $\Lambda=\left\{\mathbf{e}_{1}, \ldots, \mathbf{e}_{m}\right\}$ is the minimal point attractor of the $\operatorname{RDS} \varphi$.

Proof. The measurability and invariance properties of a point attractor clearly hold for $\Lambda$. Further, each point attractor has to contain $\Lambda$, since each point in $\Lambda$ is invariant under every $V \in \mathcal{V}$. Therefore, it only remains to show that for each $\mathbf{x} \in S^{m-1}$, we have

$$
\lim _{n \rightarrow \infty} d\left(\varphi\left(n, \vartheta_{-n} \omega\right)(\mathbf{x}), \Lambda\right)=0 \quad \mathbb{P} \text {-a.s. }
$$

If we replace 'P $\mathrm{P}$-a.s.' by 'in probability', then the result follows immediately from Theorem 3.1. In order to infer almost sure convergence from convergence in probability, it suffices to show that convergence in probability happens sufficiently quickly. In fact, 
thanks to the first Borel-Cantelli lemma, it suffices to prove that for each $\varepsilon>0$, we have

$$
\sum_{n=1}^{\infty} \mathbb{P}\left(T_{n} \circ \cdots \circ T_{1}(\mathbf{x}) \in U_{\varepsilon}\right)<\infty .
$$

Observe that Propositions 3.2 and 3.3 together show that the summands converge to zero exponentially quickly and therefore the assertion follows.

\section{A. Appendix}

Proposition A.1. Consider a real-valued process $\left(Y_{n}\right)_{n \in \mathbb{N}_{0}}$ that is in $\mathcal{L}^{1}(\mathbb{P})$ and adapted to a filtration $\left(\mathcal{F}_{n}\right)_{n \in \mathbb{N}_{0}}$ such that for some $a \in \mathbb{R}$ and $A, B>0$, we have that for all $n \in$ $\mathbb{N}_{0}$ :

(1) $\mathbb{E}\left[Y_{n+1} \mid \mathcal{F}_{n}\right] \geq Y_{n}+A$; and

(2) $\mathbb{E}\left[\left(Y_{n+1}-\mathbb{E}\left[Y_{n+1} \mid \mathcal{F}_{n}\right]\right)^{2} \mid \mathcal{F}_{n}\right] \leq B$

on $\left\{Y_{n} \geq a\right\} \mathbb{P}$-almost surely. Then

$$
\mathbb{P}\left(\liminf _{n \rightarrow \infty} \frac{1}{n} Y_{n} \geq A \mid \forall n \in \mathbb{N}: Y_{n} \geq a\right)=1 .
$$

Moreover, for every $\theta>0$ and every $b \in \mathbb{R}$, there exists an $S \in \mathbb{R}$ such that

$$
\mathbb{P}\left(\forall n \in \mathbb{N}: Y_{n}>b \mid \mathcal{F}_{0}\right) \geq 1-\theta
$$

$\mathbb{P}$-almost surely on $\left\{Y_{0} \geq S\right\}$.

Proof. The proof of (A.2) follows an idea of Rajchman used to prove a strong law of large numbers; see [22, Theorem 2.14]. A similar result with stronger assumptions is given in [29, Lemma 2.6].

We begin with the proof of the first statement and define $\tau:=\left\{n \in \mathbb{N} \mid Y_{n}<a\right\}$ as the first time our process jumps below the level $a$.

We will want to apply Theorem 2.19 from [19] to the sequence $\left(\left(Y_{n+1}-\mathbb{E}\left[Y_{n+1} \mid\right.\right.\right.$ $\left.\left.\left.\mathcal{F}_{n}\right]\right) \mathbb{1}_{\{\tau>n\}}\right)_{n \in \mathbb{N}_{0}}$.

Therefore, let $\Xi$ be a random variable such that $\mathbb{P}(\Xi \leq 1)=0$ and $\mathbb{P}(\Xi>x)=1 / x^{2}$ for $x>1$. Then $\mathbb{E}\left[\Xi \log ^{+} \Xi\right]<\infty$ and, since

$$
\begin{aligned}
\mathbb{P}\left(\left|Y_{n+1}-\mathbb{E}\left[Y_{n+1} \mid \mathcal{F}_{n}\right]\right| \mathbb{1}_{\{\tau>n\}}>x\right) & \leq\left(\mathbb{E}\left[\left(Y_{n+1}-\mathbb{E}\left[Y_{n+1} \mid \mathcal{F}_{n}\right]\right)^{2} \mathbb{1}_{\{\tau>n\}}\right] \frac{1}{x^{2}}\right) \wedge 1 \\
& \leq\left(B \frac{1}{x^{2}}\right) \wedge 1=(B \vee 1) \mathbb{P}(\Xi>x)
\end{aligned}
$$

for all $x>0$ and $n \in \mathbb{N}_{0}$, the assumptions of the theorem hold and we have

$$
\begin{aligned}
& \frac{1}{n} \sum_{i=1}^{n}\left(Y_{i+1}-\mathbb{E}\left[Y_{i+1} \mid \mathcal{F}_{i}\right]\right) \mathbb{1}_{\{\tau>i\}} \\
& \quad=\frac{1}{n} \sum_{i=1}^{n}\left(\left(Y_{i+1}-\mathbb{E}\left[Y_{i+1} \mid \mathcal{F}_{i}\right]\right) \mathbb{1}_{\{\tau>i\}}-\mathbb{E}\left[\left(Y_{i+1}-\mathbb{E}\left[Y_{i+1} \mid \mathcal{F}_{i}\right]\right) \mathbb{1}_{\{\tau>i\}} \mid \mathcal{F}_{i}\right]\right) \stackrel{n \rightarrow \infty}{\longrightarrow} 0
\end{aligned}
$$

$\mathbb{P}$-almost surely. 
Now observe that

$$
\begin{aligned}
& \liminf _{n \rightarrow \infty} \frac{1}{n} Y_{n \wedge \tau}= \liminf _{n \rightarrow \infty} \frac{1}{n} \sum_{i=1}^{n}\left(Y_{i}-Y_{i-1}\right) \mathbb{1}_{\{\tau>i-1\}} \\
&= \liminf _{n \rightarrow \infty} \frac{1}{n} \sum_{i=1}^{n}\left(\left(Y_{i}-Y_{i-1}\right) \mathbb{1}_{\{\tau>i-1\}}\right. \\
&\left.\quad-\mathbb{E}\left[\left(Y_{i}-Y_{i-1}\right) \mathbb{1}_{\{\tau>i-1\}} \mid \mathcal{F}_{i-1}\right]+\mathbb{E}\left[\left(Y_{i}-Y_{i-1}\right) \mathbb{1}_{\{\tau>i-1\}} \mid \mathcal{F}_{i-1}\right]\right) \\
&= \liminf _{n \rightarrow \infty} \frac{1}{n} \sum_{i=1}^{n}\left(\left(Y_{i}-\mathbb{E}\left[Y_{i} \mid \mathcal{F}_{i-1}\right]\right) \mathbb{1}_{\{\tau>i-1\}}\right. \\
&\left.+\mathbb{E}\left[\left(Y_{i}-Y_{i-1}\right) \mathbb{1}_{\{\tau>i-1\}} \mid \mathcal{F}_{i-1}\right]\right) \\
& \stackrel{(\text { A.3) }}{=} \liminf _{n \rightarrow \infty} \frac{1}{n} \sum_{i=1}^{n} \mathbb{E}\left[\left(Y_{i}-Y_{i-1}\right) \mathbb{1}_{\{\tau>i-1\}} \mid \mathcal{F}_{i-1}\right] \\
&=\liminf _{n \rightarrow \infty} \frac{1}{n} \sum_{i=1}^{n} \underbrace{\left(\mathbb{E}\left[Y_{i} \mid \mathcal{F}_{i-1}\right]-Y_{i-1}\right)}_{\geq A} \mathbb{1}_{\{\tau>i-1\}} \\
& \geq \liminf _{n \rightarrow \infty} \frac{n \wedge \tau}{n} A
\end{aligned}
$$

and therefore

$$
\liminf _{n \rightarrow \infty} \frac{1}{n} Y_{n} \geq A \quad \text { on }\{\tau=\infty\}
$$

which proves the first statement.

To prove the second statement, we start by considering a process $\left(\bar{Y}_{n}\right)_{n \in \mathbb{N}}$ with the same properties as $\left(Y_{n}\right)_{n \in \mathbb{N}_{0}}$, but without the restriction on the size of the predecessor, i.e. such that for all $n \in \mathbb{N}_{0}$ :

(1') $\mathbb{E}\left[\bar{Y}_{n+1} \mid \mathcal{F}_{n}\right] \geq \bar{Y}_{n}+A$; and

(2') $\mathbb{E}\left[\left(\bar{Y}_{n+1}-\mathbb{E}\left[\bar{Y}_{n+1} \mid \mathcal{F}_{n}\right]\right)^{2} \mid \mathcal{F}_{n}\right] \leq B$

$\mathbb{P}$-almost surely.

With this, define

$$
h_{i+1}:=\bar{Y}_{i+1}-\mathbb{E}\left[\bar{Y}_{i+1} \mid \mathcal{F}_{i}\right], \quad S_{i}:=\sum_{j=1}^{i} h_{j}
$$

for all $i \in \mathbb{N}_{0}$. Note that due to $\left(2^{\prime}\right)$ we know that

$$
\mathbb{E}\left[h_{i}^{2} \mid \mathcal{F}_{0}\right] \leq B \quad \text { and } \quad \mathbb{E}\left[h_{i} h_{j} \mid \mathcal{F}_{0}\right]=0
$$

hold for every $i, j \in \mathbb{N}_{0}, i \neq j \mathbb{P}$-almost surely. For arbitrary constants $c_{1} \geq c_{2} \geq 0$ and $\alpha_{1}>\alpha_{2}>0$, we can then estimate

$$
\begin{aligned}
& \mathbb{P}(\exists\left.m \in \mathbb{N}: S_{m} \leq-c_{1}-\alpha_{1} m \mid \mathcal{F}_{0}\right) \\
& \leq \mathbb{P}\left(\exists n \in \mathbb{N}: S_{n^{2}} \leq-c_{2}-\alpha_{2} n^{2} \mid \mathcal{F}_{0}\right) \\
& \quad+\mathbb{P}\left(\exists n \in \mathbb{N} \exists m \in\left[n^{2},(n+1)^{2}-1\right]: S_{m}-S_{n^{2}} \leq-\left(c_{1}-c_{2}\right)-\left(\alpha_{1}-\alpha_{2}\right) n^{2} \mid \mathcal{F}_{0}\right)
\end{aligned}
$$




$$
\begin{aligned}
\leq & \sum_{n \in \mathbb{N}} \mathbb{P}\left(S_{n^{2}} \leq-c_{2}-\alpha_{2} n^{2} \mid \mathcal{F}_{0}\right) \\
& +\sum_{n \in \mathbb{N}} \sum_{m=n^{2}} \underbrace{\mathbb{P}\left(S_{m}-S_{n^{2}} \leq-\left(c_{1}-c_{2}\right)-\left(\alpha_{1}-\alpha_{2}\right) n^{2} \mid \mathcal{F}_{0}\right)}_{\leq\left(\sum_{i=n^{2}+1}^{m} \mathbb{E}\left[h_{i}^{2} \mid \mathcal{F}_{0}\right]\right) /\left(\left(\left(c_{1}-c_{2}\right)+\left(\alpha_{1}-\alpha_{2}\right) n^{2}\right)^{2}\right)} \\
\leq & \underbrace{\sum_{n \in \mathbb{N}} \frac{B n^{2}}{\left(c_{2}+\alpha_{2} n^{2}\right)^{2}}}_{=: f\left(c_{2}, \alpha_{2}\right)}+\underbrace{\sum_{n \in \mathbb{N}} \frac{B n(2 n+1)}{\left(\left(c_{1}-c_{2}\right)+\left(\alpha_{1}-\alpha_{2}\right) n^{2}\right)^{2}}}_{=: g\left(c_{1}-c_{2}, \alpha_{1}-\alpha_{2}\right)}
\end{aligned}
$$

$\mathbb{P}$-almost surely, where $\lim _{c_{2} \rightarrow \infty} f\left(c_{2}, \alpha_{2}\right)=0$ and $\lim _{c \rightarrow \infty} g\left(c, \alpha_{1}-\alpha_{2}\right)=0$. This means that for every $\theta>0$ (and every choice of $\alpha_{1}>\alpha_{2}>0$ ) choosing $c_{2}$ large enough for $f\left(c_{2}, \alpha_{2}\right) \leq \theta / 2$ and then $c_{1}$ large enough such that $g\left(c_{1}-c_{2}, \alpha_{1}-\alpha_{2}\right)<\theta / 2$ we have

$\mathbb{P}\left(\exists m \in \mathbb{N}: S_{m} \leq-c_{1}-\alpha_{1} m \mid \mathcal{F}_{0}\right) \leq f\left(c_{2}, \alpha_{2}\right)+g\left(c_{1}-c_{2}, \alpha_{1}-\alpha_{2}\right) \leq \theta$

$\mathbb{P}$-almost surely. Using $\alpha_{1}:=A$, we obtain the following for our process $\left(\bar{Y}_{n}\right)_{n \in \mathbb{N}_{0}}$ : for every $\theta>0$ and every point $b$ choosing $S:=c_{1}+b$ for $c_{1}$ as above we get that on $\left\{\bar{Y}_{0} \geq S\right\}$

$$
\begin{aligned}
\mathbb{P}\left(\exists m \in \mathbb{N}: \bar{Y}_{m} \leq b \mid \mathcal{F}_{0}\right) & =\mathbb{P}\left(\exists m \in \mathbb{N}: \bar{Y}_{0}+\sum_{i=1}^{m}\left(\bar{Y}_{i}-\bar{Y}_{i-1}\right) \leq b \mid \mathcal{F}_{0}\right) \\
& \leq \mathbb{P}\left(\exists m \in \mathbb{N}: S+\sum_{i=1}^{m}\left(\bar{Y}_{i}-\bar{Y}_{i-1}\right) \leq b \mid \mathcal{F}_{0}\right) \\
& \leq \mathbb{P}\left(\exists m \in \mathbb{N}: \sum_{i=1}^{m}\left(\bar{Y}_{i}-\bar{Y}_{i-1}\right) \leq b-S \mid \mathcal{F}_{0}\right) \\
& \leq \mathbb{P}(\exists m \in \mathbb{N}: \sum_{i=1}^{m} h_{i} \leq b-S-\sum_{i=1}^{\sum_{\geq A}^{m}} \underbrace{\mathbb{E}\left[\overline{\mathcal{F}}_{0}\right)}_{\left.\geq \bar{Y}_{i}-\bar{Y}_{i-1} \mid \mathcal{F}_{i-1}\right]} \\
& \leq \mathbb{P}(\exists m \in \mathbb{N}: \sum_{i=1}^{m} h_{i} \leq \underbrace{b-S}_{=-c_{1}}-m \underbrace{A}_{=\alpha_{1}} \mid \mathcal{F}_{0}) \leq \theta,
\end{aligned}
$$

which means that for every $\theta>0$ and $b \in \mathbb{R}$ we can find an $S$ such that

$$
\mathbb{P}\left(\forall m \in \mathbb{N}: \bar{Y}_{m}>b \mid \mathcal{F}_{0}\right) \geq 1-\theta \quad \text { on }\left\{\bar{Y}_{0} \geq S\right\} .
$$

Coming back to $\left(Y_{n}\right)_{n \in \mathbb{N}_{0}}$, use it to define such a process $\left(\bar{Y}_{n}\right)_{n \in \mathbb{N}_{0}}$ through $\bar{Y}_{0}:=Y_{0}$ and

$$
\bar{Y}_{n+1}-\bar{Y}_{n}:= \begin{cases}Y_{n+1}-Y_{n} & \text { if } \tau>n, \\ A & \text { otherwise. }\end{cases}
$$

This process has the stronger properties $\left(1^{\prime}\right)$ and $\left(2^{\prime}\right)$ and, since we also have $\left\{\forall n \in \mathbb{N}_{0}\right.$ : $\left.\bar{Y}_{n} \geq a\right\}=\left\{\forall n \in \mathbb{N}_{0}: Y_{n} \geq a\right\}$, the above observation yields the second statement. 
Acknowledgements. The authors would like to thank the referee for his/her comments and suggestions that contributed to improve this paper. Furthermore, the first-named author (U.U.J.) thanks the IMU Berlin Einstein Foundation Program (EFP) and the Berlin Mathematical School (BMS) for a scholarship and for supporting his visit to the Technische Universität (TU) Berlin, Germany and the Programme Erasmus Mundus Action 2 (EMA2) Marco XXI for a scholarship and for supporting his visit to the University of Santiago de Compostela (USC), Spain. He also thanks the TU Berlin and USC for the kind hospitality and for providing all facilities.

\section{REFERENCES}

[1] L. Arnold. Random Dynamical Systems. Springer, Berlin, 1998.

[2] S. N. Bernstein. The solution of a mathematical problem related to the theory of heredity. Uchn. Zapiski NI Kaf. Ukr. Otd. Mat.(1) (1924), 83-115 (in Russian).

[3] I. Chueshov and M. Scheutzow. On the structure of attractors and invariant measures for a class of monotone random systems. Dyn. Syst. 19 (2004), 127-144.

[4] H. Crauel. Global random attractors are uniquely determined by attracting deterministic compact sets. Ann. Mat. Pura Appl. (4) 176 (1999), 57-72.

[5] H. Crauel. Random point attractors versus random set attractors. J. Lond. Math. Soc. (2) 63 (2001), 413-427.

[6] H. Crauel, G. Dimitroff and M. Scheutzow. Criteria for strong and weak random attractors. J. Dynam. Differential Equations 21 (2009), 233-247.

[7] H. Crauel and F. Flandoli. Attractors for random dynamical systems. Probab. Theory Related Fields 100 (1994), 365-393.

[8] N. N. Ganikhodzhaev. An application of the theory of Gibbs distributions to mathematical genetics. Dokl. Math. 61(3) (2000), 321-323.

[9] N. N. Ganikhodzhaev. The random models of heredity in the random environments. Dokl. Akad. Nauk $R U z .(12)$ (2001), 6-8 (in Russian).

[10] N. N. Ganikhodjaev, R. N. Ganikhodjaev and U. U. Jamilov. Quadratic stochastic operators and zero-sum game dynamics. Ergod. Th. \& Dynam. Sys. (2014), doi:10.1017/etds.2013.109.

[11] N. N. Ganikhodjaev, U. U. Jamilov and R. T. Mukhitdinov. On non-ergodic transformations on $S^{3}$. J. Phys.: Conf. Ser. 435 (2013), 012005, doi:10.1088/1742-6596/435/1/012005.

[12] N. N. Ganikhodjaev, U. U. Jamilov and R. T. Mukhitdinov. Non-ergodic quadratic operators of bisexual population. Ukrainian Math. J. 65(6) (2013), 1152-1160.

[13] N. N. Ganikhodjaev and D. V. Zanin. On a necessary condition for the ergodicity of quadratic operators defined on the two-dimensional simplex. Russian Math. Surveys 59(3) (2004), 571-572.

[14] N. N. Ganikhodjaev and D. V. Zanin. Ergodic Volterra quadratic transformations of the simplex. Preprint, 2012, arXiv:1205.3841 (in Russian).

[15] R. N. Ganikhodzhaev. Quadratic stochastic operators, Lyapunov functions, and tournaments. Sb. Math. 76(2) (1993), 489-506.

[16] R. N. Ganikhodzhaev. Map of fixed points and Lyapunov functions for a class of discrete dynamical systems. Math. Notes 56(5) (1994), 1125-1131.

[17] R. N. Ganikhodzhaev and D. B. Eshmamatova. Quadratic automorphisms of a simplex and the asymptotic behavior of their trajectories. Vladikavkaz. Mat. Zh. 8(2) (2006), 12-28 (in Russian).

[18] R. N. Ganikhodzhaev, F. M. Mukhamedov and U. A. Rozikov. Quadratic stochastic operators and processes: results and open problems. Infin. Dimens. Anal. Quantum Probab. Relat. Top. 14(2) (2011), 279-335.

[19] P. Hall and C. C. Heyde. Martingale Limit Theory and its Application. Academic Press, New York, 1980.

[20] H. Kesten. Quadratic transformations: a model for population growth I. Adv. Appl. Probab. 2(1) (1970), $1-82$.

[21] H. Kesten. Quadratic transformations: a model for population growth. II. Adv. Appl. Probab. 2(2) (1970), 179-228.

[22] U. Krengel. Einführung in die Wahrscheinlichkeitstheorie und Statistik, 6. Aufl. Vieweg, Braunschweig, 2002. 
[23] Yu. I. Lyubich. Mathematical Structures in Population Genetics (Biomathematics, 22). Springer, Berlin, 1992.

[24] F. Mukhamedov, H. Akin and S. Temir. On infinite dimensional quadratic Volterra operators. J. Math. Anal. Appl. 310 (2005), 533-556.

[25] U. A. Rozikov and U. U. Jamilov. F-quadratic stochastic operators. Math. Notes 83(4) (2008), 554-559.

[26] U. A. Rozikov and U. U. Jamilov. The dynamics of strictly non-Volterra quadratic stochastic operators on the two-dimensional simplex. Sb. Math. 200(9) (2009), 1339-1351.

[27] U. A. Rozikov and U. U. Jamilov. Volterra quadratic stochastic of a two-sex population. Ukrainian Math. J. 63(7) (2011), 1136-1153.

[28] M. Scheutzow. Comparison of various concepts of a random attractor: a case study. Arch. Math. 78 (2002), 233-240.

[29] M. Scheutzow and D. Steinsaltz. Chasing balls through martingale fields. Ann. Probab. 30 (2002), 2046-2080.

[30] S. M. Ulam. A Collection of Mathematical Problems. Interscience, New York-London, 1960.

[31] S. S. Vallander. On the limit behavior of iteration sequences of certain quadratic transformations. Sovrem. Mat. Dokl. 13 (1972), 123-126.

[32] M. I. Zakharevich. On the behavior of trajectories and the ergodic hypothesis for quadratic mappings of a simplex. Russian Math. Surveys 33(6) (1978), 265-266. 